\title{
The Structure of Space-Time Transformations
}

\author{
H. J. Borchers and G. C. Hegerfeldt \\ Institut für Theoretische Physik, Universität Göttingen, Göttingen, Germany
}

Received May 15, 1972

\begin{abstract}
Let $T$ be a one-to-one mapping of $n$-dimensional space-time $M$ onto itself. If $T$ maps light cones onto light cones and $\operatorname{dim} M \geqq 3$, it is shown that $T$ is, up to a scale factor, an inhomogeneous Lorentz transformation. Thus constancy of light velocity alone implies the Lorentz group (up to dilatations). The same holds if $T$ and $T^{-1}$ preserve $(x-y)^{2}>0$. This generalizes Zeeman's Theorem. It is then shown that if $T$ maps lightlike lines onto (arbitrary) straight lines and if $\operatorname{dim} M \geqq 3$, then $T$ is linear. The last result can be applied to transformations connecting different reference frames in a relativistic or nonrelativistic theory.
\end{abstract}

\section{Introduction}

Let $M$ denote $n$-dimensional space-time (Minkowski space). It is an affine space of $n$-tuples $x=\left(x_{0}, \ldots, x_{n-1}\right)$, where $x_{0}=c t$. We denote by $x^{2}$ the quadratic form ${ }^{1}$

$$
x^{2} \equiv x_{0}^{2}-\sum_{1}^{n-1} x_{i}^{2} .
$$

Zeeman [1] has shown for $\operatorname{dim} M \geqq 3$ that a mapping $T$ of $M$ onto $M$ is an orthochronous Lorentz transformation ${ }^{2}$ times a dilatation plus a translation if $T$ and $T^{-1}$ preserve the relation

$$
\left\{(y-x)^{2}>0 \text { and } x_{0}<y_{0}\right\}
$$

or the relation

$$
\left\{(y-x)^{2}=0 \text { and } x_{0}<y_{0}\right\} .
$$

Since the direction of time plays no particular role in quantum field theory and since moreover time reversal is an important symmetry one should drop time order preservation, which, in fact, is quite a strong continuity condition ${ }^{3}$. Thus, instead of Eqs. (1.2) or (1.3) we take as

${ }^{1}$ As usual we call two points $x$ and $y$ timelike if $(y-x)^{2}>0$, spacelike if $(y-x)^{2}<0$, and lightlike if $(y-x)^{2}=0$. The light cone in $x$ consists of all $y$ with $(y-x)^{2}=0$.

${ }^{2}$ I.e., linear maps of $M$ which preserve the form (1.1) and preserve time orientation.

${ }^{3}$ It follows immediately from the preservation of Eq. (1.2) that $T$ is continuous. Indeed, preservation of (1.2) means that $T$ is continuous with respect to the associated order topology which, however, coincides for finite-dimensional spaces with Euclidean topology as already noted in [5]. 
preserved relations only

or

$$
(x-y)^{2}>0
$$

$$
(x-y)^{2}=0 \text {. }
$$

The latter just expresses constancy of light velocity. In Section 2 we will prove

Theorem 1. Let $\operatorname{dim} M \geqq 3$ and $T$ be a $1-1$ map of $M$ onto $M$. Then $T$ and $T^{-1}$ preserve the relation $(x-y)^{2}>0$ if and only if they preserve the relation $(x-y)^{2}=0$. The group of all such maps is generated by

(i) the full Lorentz group (including time reversal),

(ii) translations of $M$,

(iii) dilations (multiplication by a scalar).

Preservation of $(x-y)^{2}=0$ by $T$ and $T^{-1}$ simply means that light cones are mapped onto light cones. Thus constancy of light velocity $c$ alone implies the Poincaré group ${ }^{4}$ (up to dilatations)!

The main statement of Theorem 1 is that $T$ is affine ("linear"), and one wonders whether linearity also holds in the nonrelativistic case. Of course, $c$ is no longer constant in all reference frames. If light cones are mapped onto light cones then lightlike lines ${ }^{5}$ are mapped onto lightlike lines since any such line is the intersection of two light cones.

In the relativistic as well as nonrelativistic case the worldlines of light rays (photons) should be mapped onto straight lines. It suffices to consider light sources at rest ${ }^{6}$ to be able to apply the next result.

Theorem 2. Let $\operatorname{dim} M \geqq 3$, and let $T$ be a 1-1 map of $M$ onto $M$ which maps lightlike lines onto (arbitrary) straight lines. Then $T$ is linear.

Remarks. (i) Theorem 1 can be derived from Theorem 2 in a straightforward way. Our proofs of both theorems are, however, more or less independent (Sections 2 and 3). For $\operatorname{dim} M=2$ the theorems do not hold.

(ii) For linearity, the rotational symmetry of the light cone ("isotropy of light velocity") is not crucial. It suffices that its boundary is sufficiently smooth, i.e., sufficiently smooth directional dependence of light velocity.

(iii) Our results severely limit the form of any physical theory which retains straight lines for light rays and which has no distinguished reference frames. For then the laws of Physics have to be form-invariant under transformations connecting different reference frames.

${ }^{4}$ I.e., the inhomogeneous Lorentz group, generated by (i) and (ii).

${ }^{5} \mathrm{~A}$ (straight) line is called timelike, spacelike, or lightlike if any two points on it are timelike, spacelike, or lightlike, respectively.

${ }^{6}$ For these the light velocity is also $c$ in the nonrelativistic case. But in another reference frame the light velocity becomes direction dependent. 
Notation. $C_{a}$ denotes the light cone in a, $C_{a}=\left\{x \in M ;(x-a)^{2}=0\right\}$. The interior ${ }^{7} \hat{C}_{a}$ of a light cone consists of all points timelike to a, $\hat{C}_{a}=\left\{x ;(x-a)^{2}>0\right\} . \hat{C}_{a}^{+}$denotes the positive cone $\hat{C}_{a}^{+}=\left\{x ;(x-a)^{2}>0\right.$, $\left.x_{0}>a_{0}\right\}$, and $C_{a}^{-}$the negative cone. The image under a map will be denoted by a dash, e.g., $x^{\prime} \equiv T(x)$.

\section{Constancy of Light Velocity Implies the Poincaré Group}

The results of this section are based on the following observation.

Lemma 2.1. Let $W$ be a lightlike line in $M, W=\{x=\lambda w+a$; $-\infty<\lambda<\infty\}$ say, where $w^{2}=0$, and consider the hyperplane $H_{W}$ $\equiv\{x ; w \cdot(x-a)=0\}$, which contains $W$. Then $H_{W}$ is tangent to every light cone with vertex in $W$, and the union of all these cones is $M \backslash\left(H_{W} \backslash W\right)^{8}$.

Proof. Let $C_{\tau} \equiv\left\{x ;(x-\tau w-a)^{2}=0\right\}$, the cone with vertex in $\tau w+a$. Let $y \notin H_{W}$. Then $y \in C_{\tau}$ with $\tau=(y-a)^{2} / 2 w \cdot(y-a)$. Now let $y \in H_{W}$ $\cap \bigcup_{\tau} C_{\tau}$. Then

$$
0=\left(y-\tau_{0} w-a\right)^{2}=(y-a)^{2}-2 \tau_{0} w \cdot(y-a)=(y-a)^{2}
$$

implies $y \in \bigcap_{\tau} C_{\tau}=W$. Q.E.D.

The next corollary reduces Theorem 1 to the result of Zeeman [1]. Further below, however, we will give a direct derivation based on Lemma 2.1.

Corollary 2.2. Let $\operatorname{dim} M>2$, and let $T$ be a 1-1-mapping of $M$ onto itself. Then the following statements are equivalent.

(i) $T$ maps light cones onto light cones, i.e., $T$ and $T^{-1}$ preserve $(x-y)^{2}=0$.

(ii) $T$ maps the interior of light cones onto the interior of light cones, i.e., $T$ and $T^{-1}$ preserve $(x-y)^{2}>0$.

(iii) $T$ and $T^{-1}$ preserve the relation $\left\{(x-y)^{2}>0, x_{0}<y_{0}\right\}$ or carry it over in $\left\{\left(x^{\prime}-y^{\prime}\right)^{2}>0, x_{0}^{\prime}>y_{0}^{\prime}\right\}$, i.e., $T$ fulfills (ii) and preserves or reverses time order.

(iv) $T$ fulfills (i) and preserves or reverses time order.

Proof. If $T$ fulfills (i), it maps a lightlike line $W$ onto a lightlike line $W^{\prime}$ and hence, by Lemma $2.1, M \backslash\left(H_{W} \backslash W\right)$ onto $M \backslash\left(H_{W^{\prime}} \backslash W^{\prime}\right)$. Hence $H_{W}$ is mapped onto $H_{W^{\prime}}$. If $C_{a}$ is a given light cone and $\hat{C}_{a}$ its interior, then $\cup\left\{H_{W} ; W \subset C_{a}\right\}=M \backslash \hat{C}_{a}$ since $w \neq 0, w^{2}=0$ and $w \cdot(x-a)=0$

${ }^{7}$ This is an abuse of language. $C_{a}$ is the boundary of $\hat{C}_{a}$.

${ }^{8}$ I.e., every point in this set can be reached by a lightlike line from some point of $W$. Note that, for $\operatorname{dim} M=2$, the hyperplane coincides with $W$ and hence $M \backslash\left(H_{W} \backslash W\right)=M$. 
immediately implies $(x-a)^{2} \leqq 0$; conversely, for given $x$ with $(x-a)^{2}=0$ there is always a lightlike $w \neq 0$ with $w \cdot(x-a)=0$ if $\operatorname{dim} M \geqq 3$. Hence $M \backslash \hat{C}_{a}$ is mapped onto $M \backslash \hat{C}_{a^{\prime}}$ and thus $\hat{C}_{a}$ onto $\hat{C}_{a^{\prime}}$. Hence (i) $\Rightarrow$ (ii).

To show (ii) $\Rightarrow\left(\right.$ iii) ${ }^{9}$, we first assume that there is an $a \in M$ and $b \in \hat{C}_{a}^{+}$such that $b^{\prime} \in \hat{C}_{a^{\prime}}^{+}$. Then $\hat{C}_{b}^{-} \supset \hat{C}_{a}^{-}$and $\hat{C}_{b^{\prime}}^{-} \supset \hat{C}_{a^{\prime}}^{-}$. Now let $y \in \hat{C}_{a}^{+}$. If $y \notin \hat{C}_{b}$, then $y^{\prime} \notin \hat{C}_{b^{\prime}} \supset \hat{C}_{a^{\prime}}^{-}$and thus $y^{\prime} \in C_{a^{\prime}}^{+}$. If $y \in \hat{C}_{b}$, there is a $z \in \hat{C}_{a}^{+} \backslash \hat{C}_{b}$ such that $y \notin C_{z}$. Then $\hat{C}_{z} \supset \hat{C}_{z}^{-} \supset \hat{C}_{a}^{-}$and $z^{\prime} \in C_{a^{\prime}}^{+}$implies $y^{\prime} \notin C_{z^{\prime}} \supset \hat{C}_{a^{\prime}}^{-}$. Thus $y^{\prime} \in \hat{C}_{a^{\prime}}^{+}$. Hence $\hat{C}_{a}^{+}$is mapped into $\hat{C}_{a^{\prime}}^{+}$. Hence there is a $c \in \hat{C}_{a}^{-}$with $c^{\prime} \in \hat{C}_{a^{\prime}}^{-}$and the same argument gives $T\left(\hat{C}_{a}^{-}\right) \subset \hat{C}_{a^{\prime}}^{-}$. Hence $\hat{C}_{a}^{+}$is mapped onto $\hat{C}_{a^{\prime}}^{+}$, and $\hat{C}_{a}^{-}$onto $\hat{C}_{a^{\prime}}^{-}$. Since two light cones always intersect, one gets the same for all $\hat{C}_{x}^{ \pm}$. If there is no $a$ and $b$ with the above property, then $T\left(\hat{C}_{a}^{+}\right)=\hat{C}_{a^{\prime}}^{-}$for all $a$, since otherwise there would be an $a \in M$ and $b \in \hat{C}_{a}^{-}$with $b^{\prime} \in \hat{C}_{a^{\prime}}^{-}$, and by the same argument $T\left(\hat{C}_{a}^{-}\right) \subset C_{a^{\prime}}^{-}$, contradicting $T\left(\hat{C}_{a^{\prime}}\right)=\hat{C}_{a^{\prime}}$.

(iii) $\Rightarrow$ (iv) is very simple and follows directly from Lemma 1 of [1] since, if $\tau$ denotes time reversal, either $T$ and $T^{-1}$ or $\tau T$ and $(\tau T)^{-1}$ preserve $\left\{(x-y)^{2}, x_{0}<y_{0}\right\}$. (iv) $\Rightarrow$ (i) is trivial. Q.E.D.

We will now give a fairly simple proof of Theorem 1 without using Zeeman's result. We first note some simple properties of the tangential hyperplane $H_{W}$.

Lemma 2.3. $H_{W}$ contains only spacelike and lightlike lines, and the latter are all parallel to $W$.

Proof. We use the notation of the proof of Lemma 2.1. Let $W_{1} \subset H_{W}$, $W_{1}=\left\{x=\lambda w_{1}+b ;-\infty<\lambda<\infty\right\}$. After a parallel displacement, we can assume $b=a$. If $W_{1}$ is lightlike, $w \cdot\left(\lambda w_{1}+a-a\right)=0$ implies by Eq. (2.1), with $\lambda=\lambda w_{1}+a$, that $W_{1} \subset \bigcap C_{\tau}=W$. If $W_{1}$ were timelike, then $w \cdot w_{1}=0$, but $w_{1}^{2}>0$ and $w^{2}=0$; this is impossible (alternatively, $H_{W}$ does not contain points of $\hat{C}_{\tau}$ ). Q.E.D.

Lemma 2.4. Every spacelike line $W$ is the intersection of suitable hyperplanes $H_{W_{i}}, i=1, \ldots, n-1=\operatorname{dim} M-1$.

Proof. We can assume $W=\{x=\lambda w ;-\infty<\lambda<\infty\}, w^{2}<0$. Then there are $n-1$ linearly independent lightlike vectors $w_{i}$ with $w \cdot w_{i}=0$. Hence, with $W_{i} \equiv\left\{\lambda w_{i}\right\}$, one has that $\bigcap_{i} H_{W_{i}}$ is a straight line which
contains $W$. Q.E.D.

By the implication (ii) $\Rightarrow$ (i) of Corollary 2.2 , which can very easily also be shown directly, it suffices to show that, if $T$ maps light cones onto light cones, then $T$ is linear and hence, up to a translation and dilatation, a Lorentz transformation. Then $T$ and $T^{-1}$ automatically preserve $(x-y)^{2}>0$.

${ }^{9}$ For $\operatorname{dim} M=2$ this is most easily seen by a diagram of which the following is an abstraction. 
Proof of Theorem 1. As remarked before, it follows from Lemma 2.1 that $H_{W}$ is mapped onto $H_{W}$ for each lightlike line $W$. Hence, by Lemma 2.4, spacelike lines are mapped onto spacelike lines ${ }^{10}$. Therefore, since each plane contains 3 spacelike directions, planes are mapped onto planes. Since every straight line is the intersection of two planes, it follows that every straight line is mapped onto a straight line. Hence, by the main theorem of projective geometry, the map $T$ is affine, $T(x)=A x+a$, where $A$ is a linear operator (matrix).

Since $x^{2}=0$ implies $(A x)^{2}=0, A$ leaves the light cone $C_{0}$ invariant and hence, from linearity $(A x)^{2}=\kappa x^{2}$ for all $x \in M$. For $\operatorname{dim} M>2$, the exterior of $C_{0}$ is connected while the interior is not. Since $T$ is affine and hence continuous, it can not map the interior onto the exterior. Hence $\kappa>0$. Thus $A=\kappa^{1 / 2} \Lambda$ where $\Lambda$ satisfies $(\Lambda x)^{2}=x^{2}$ and is thus a general Lorentz transformation. Q.E.D.

Remark. Let $\bar{M}$ denote the space obtained by adjoining to $M$ the hyperplane at infinity, and let $T$ be a 1-1-map of $\bar{M}$ onto $\bar{M}$ such that both $T$ and $T^{-1}$ map lightlike lines onto lightlike lines. Then it follows in a similar way as before that all straight lines are mapped onto straight lines. Hence $T$ is a projective transformation. Since it leaves the (extended) light cone invariant, $T$ is an element of the conformal group.

\section{Linearity for the General Case}

The proof of Theorem 2 is based on the next lemma.

Lemma 3.1. Let $\operatorname{dim} M \geqq 3$ and let $T$ be a 1-1-mapping of $M$ onto $M$ which maps lightlike lines onto straight lines. Then planes which contain two different lightlike directions are mapped onto planes.

For $\operatorname{dim} M \geqq 4$ a proof of this lemma will be given at the end of this section. The remaining case will be treated elsewhere [4]. Lemma 3.1 allows a reduction of Theorem 2 to the main theorem of projective geometry.

Proof of Theorem 2. Every line in $M$ is the intersection of two planes containing two different lightlike directions. Its image is the intersection of the two image planes, which is a straight line. Hence $T$ maps straight lines onto straight lines. Q.E.D.

${ }^{10}$ At this point one could invoke a result of [2]: If a 1-1 map $T$ maps all straight lines in a given cone and all their translates onto straight lines, then $T$ is linear. Here one can take a cone of spacelike lines. 
It remains to show Lemma 3.1. We first prove

Lemma 3.2. Under the assumptions of Lemma 3.1, let $w_{1}, w_{2}$, and $w_{3}$ be 3 linearly independent lightlike vectors, and let $S_{3}$ be the 3-dimensional subspace of $M$ spanned by them and a point $P$. Then the image of $S_{3}$ is again contained in a 3-dimensional subspace of $M$.

Proof. Let $W_{1}$ and $W_{3}$ be the lines through $P$ parallel to $w_{1}$ and $w_{3}$. Let $P_{1} \in W_{1}, P_{1} \neq P$, and let $W_{2}$ be the line through $P_{1}$ parallel to $w_{2}$. These lines and their points will be called to be of class 0 . If lines and points of class $m-1$ have been defined, we will call of class $m$ those additional lines and points obtained by drawing a lightlike line through two different points of class $m-1$.

Now, $W_{1}^{\prime}, W_{2}^{\prime}$, and $W_{3}^{\prime}$ lie in a 3-dimensional subspace, $U$ say, since four points always do. If the images of all points of class $m-1$ lie in $U$, so do their lightlike connecting lines and hence the images of all points of class $m$. We will show that every point in $S_{3}$ is of class $m \leqq 6$. This then will prove the lemma.

We denote by $W_{i}(Q)$ the parallel to $W_{i}$ through a point $Q$ and put $H_{i} \equiv H_{W_{i}} \cap S_{3}$ where $H_{W_{i}}$ is the tangent hyperplane of Lemma 2.1 containing $W_{i}$. Note that $H_{i}$ is a plane and that a lightlike line in $S_{3}$ which is not parallel to $W_{i}$ meets it in a single point.

We first show that the points in the $W_{2}-W_{3}\left(P_{1}\right)$-plane are of class $m \leqq 4$. Since $W_{3}$ meets $H_{2}$ in a single point, there are points $P_{2} \in W_{2}$ and

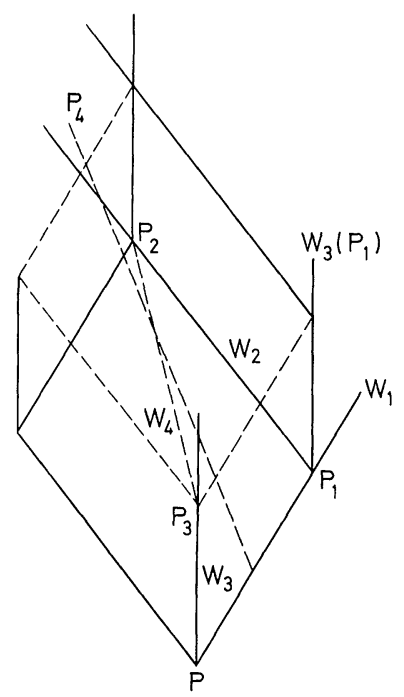

Fig. 1. Proof of Lemma 3.2 
$P_{3} \in W_{3}, P_{2} \neq P_{1}$ and $P_{3} \neq P$, which can be connected by a lightlike line, $W_{4}$ say. $W_{4}$ is of class 1 . Since $W_{4}$ is not parallel to $W_{1}$, it meets $H_{1}$ in a single point.

Hence, except for a single point, every point on $W_{4}$ can be connected by a lightlike line with a point ${ }^{11}$ on $W_{1}$, and vice versa. These lines meet the $W_{2}-W_{3}\left(P_{1}\right)$-plane, each in a single point, with the exception of $W_{2}$ and $W_{3}$. From these points of class $m \leqq 2$ we can choose two, $P_{4}$ and $P_{5}$ say, such that $\overrightarrow{P_{4} P_{5}}$ is not parallel to $W_{3}$. Then $W_{3}\left(P_{4}\right)$ and $W_{3}\left(P_{5}\right)$ do not coincide, both meet $W_{2}$, and hence are of class $m \leqq 3$. The parallels to $W_{2}$ through them are of class $m \leqq 4$ and cover the $W_{2}-W_{3}\left(P_{1}\right)$-plane.

It follows that the points of the $W_{3}-W_{3}\left(P_{2}\right)$-plane are of class $m \leqq 5$. Finally, an arbitrary $Q \in S_{3} \backslash W_{3}\left(P_{2}\right)$ lies on $W_{1}(Q)$ which meets the $W_{2}-W_{3}\left(P_{1}\right)$ - and $W_{3}-W_{3}\left(P_{2}\right)$-plane in distinct points; hence $Q$ is of class $m \leqq 2^{12}$. Q.E.D.

Proof of Lemma 3.1. Let $E$ be a plane which contains two nonparallel lightlike lines $W_{1}$ and $W_{2}$. Let $W_{i}=\left\{x=\tau w_{i}+a_{i},-\infty<\tau<\infty\right\}$, and complete $\left\{w_{1}, w_{2}\right\}$ to a basis $\left\{w_{1}, \ldots, w_{n}\right\}$ of lightlike vectors for $M$. Let $a \in W_{1} \cap W_{2}$. For given $(\mu)=\left(\mu_{3}, \ldots, \mu_{n}\right)$, with not all $\mu_{i}$ vanishing, we consider the 3-dimensional subspace $S(\mu)$ through $a$ spanned by $w_{1}, w_{2}$, and $w(\mu)=\Sigma \mu_{i} w_{i}$. Since $S(\mu)$ always contains a third linearly independent lightlike direction, we have, by Lemma 3.2, $T(S(\mu)) \subset U(\mu)$ where $U(\mu)$ is a 3 -dimensional subspace of $M$. Since $\bigcup_{\mu} S(\mu)=M$ and since $T$ is onto, there are $(\mu)$ and $(\hat{\mu})$ such that $U(\mu) \neq U(\hat{\mu})$ if $\operatorname{dim} M>3$. From $E=S(\mu) \cap S(\hat{\mu})$ we have $T(E) \subset U(\mu) \cap U(\hat{\mu})$, and hence $E^{\prime}$ is contained in a plane. Any parallel to $W_{1}$ in $E$ is therefore mapped onto a parallel to $W_{1}^{\prime}$ since they do not meet. Therefore there is, through each point of $W_{2}^{\prime}$, a parallel to $W_{1}^{\prime}$ all of which lie in $E^{\prime}$. Since they form a plane, this proves Lemma 3.1.

Note added in proof: In a forthcoming paper [4], the authors will, by purely algebraic methods, prove the following result which is much stronger than Theorem 2 and which also covers the case of $\operatorname{dim} M=3$.

Theorem [4]: Let $L$ be a set of directions in $\mathbb{R}^{n}, n \geqq 2$, and let $\mathscr{T}_{L}$ be the set of all 1-1 mappings $T$ of $\mathbb{R}^{n}$ into itself which map every straight line parallel to $L$ onto a straight line. Then every $T \in \mathscr{T}_{L}$ is linear if and only if

(i) $L$ does not lie on a degenerate cone of second order (and, for $n=2$, contains at least 3 directions);

(ii) the subfield of $\mathbb{R}$ generated by all ratios of the form $\lambda_{i} / \lambda_{j}$, where $\Sigma \lambda_{i} h_{i} \in L$ for some fixed basis $\left\{h_{1}, \ldots, h_{n}\right\}$ in $\mathbb{R}^{n}$, coincides with $\mathbb{R}$.

${ }^{11}$ This point is unique since there are no triangles with lightlike lines. One can avoid the use of Lemma 2.1 by a very simple direct calculation.

${ }^{12}$ By a slightly different construction one can show that every point in $S_{3}$ is of class $m \leqq 3$. 
The second condition requires in particular that $L$ contains uncountably many directions. It is interesting that condition (i) is sufficient for linearity if $T$ is continuous at some point [4]. Hence in this case $1 / 2 n(n+1)-1$ suitably chosen directions will ensure linearity for $n \geqq 3$ while for $n=2$ one needs 3 .

\title{
References
}

1. Zeeman,E.C.: Causality implies the Lorentz group. J. Math. Phys. 5, 490 (1964).

2. Hegerfeldt, G.C.: The Lorentz transformations: Derivation of linearity and scale factor. Il Nuovo Cim. A 10, 257 (1972).

3. Pickert, G.: Analytische Geometrie. 6. Aufl. (Sect. 26) Leipzig: Akad. Verlagsgesellschaft 1967.

4. Borchers,H.J., Hegerfeldt,G.C.: Über ein Problem der Relativitätstheorie: Wann sind Punktabbildungen des $R^{n}$ linear? Nachr. Gött. Akad. Wiss. (to be published).

5. Flato, M., Sternheimer, D.: Remarques sur les automorphismes causals de l'espace-temps. C. R. Acad. Sc. Paris, t. 263, 935 (1966).

\author{
H. J. Borchers \\ G. C. Hegerfeldt \\ Institut für Theoretische Physik \\ Universität Göttingen \\ D-3400 Göttingen, Bunsenstr. 9 \\ Federal Republic of Germany
}

